\title{
The Influence of Basic Frequency on Concentrated Load to Analogue Probe
}

\author{
Huaibo Qiang ${ }^{1, a^{*}}$ and Qiong $W^{2, b}$ \\ ${ }^{1}$ Xi'an Technological University, School of Mechanical Engineering, Xi'an 710032, china \\ ${ }^{2}$ University of Architecture and Technology, School of Mechanical Engineering, Xi'an 710055, china \\ aqianghuaibo@126.com, b1937001533@qq.com \\ *The corresponding author
}

Keywords: Concentrated load; Basic frequency; Analogue probe; Nonlinearity

\begin{abstract}
In order to improve the response and stability on 3D analogue probe, the basic frequency was analyzed on the research of dynamic characteristics. The main components of 3D analogue probe was simplified as a concentrated load model, this paper study the effect of response characteristic and measurement accuracy on the basic frequency. Furthermore, the distribution location and influence factors on concentrated load were be researched. Results showed that it is non-linear relationship between basic frequency and load location of probe. At last, the correctness of the theoretical analysis is verified by experiment. it is an effective means to improve the dynamic performance and measurement accuracy that the frequency response characteristic should be analysis to utilize concentrated load as constraint condition.
\end{abstract}

\section{Introduction}

In the field of precision measurement, the system overall accuracy is restricted by the precision of probe and that is an important part of the measuring system. Because of the advantages of high precision and efficiency of 3D analogue probe, it was widely used in the field of precision measurement. For contact 3D analogue measuring systems, in order to improve the accuracy of measurement, and improve the dynamic stability of probe and the response characteristics, it can be necessary for basic frequency analysis in the dynamic performance study process.

In the field of study, the article[1] proposed that the uncertainty of quality was quantified using interval vector method. And a non-probabilistic method for forecasting the frequency and quality of the spring-quality system is proposed based on interval mathematical theory. The critical detached frequencies of probe were analyzed by establishing the inductance probe motion model. In order to improve the dynamic response of the probe, it were proposed to increase the probe stiffness and preload volume, reduce the quality of the active part, production of small-density materials in the article [2] [3].The relationship between modal and basic frequency should to be studied because of the need to analysis the change of basic frequency that caused by the concentrated load. Some studies suggest that the relationship between them is linear on the research[2] [3], but the author thinks the basis of this conclusion is questionable.

\section{Relationship between Concentrated Load and Basic Frequency}

The 3D analogue probe is a contact measuring instrument, and its structure is shown in figure 1.The movement of the mechanism is realized by the parallel leaf springs guide mechanism. The guiding mechanism is a critical component $t$ of the probe, and the arrangement of the other mechanism has a certain relation with it[4].Therefore, the system is abstracted as the equal section Euler-Bernoulli model that is shown in figure 2 in this study. The the parallel leaf springs guide mechanism in the model were made of four flexible sheets. Assume that the measuring force, section inertia, Young's modulus are the function of $x$ as an independent variable, that is $F^{(x)}, I(x), E(x)$. In addition, the 
probe load of the drive mechanism, the measuring mechanism, the guided mechanism etc. in the system was assumed to be the concentrated load and the coordinate position was defined.

In the study of the resonance during operation, the analogue probe is considered to be damped, that is $\zeta \neq 0$. From the simulation of the dynamic performance of the analogue probe can be obtained that the Euler-Bernoulli differential equations for forced vibration are given as follows.

$$
F(x, \theta)=\rho \frac{\partial^{2} \mathrm{Y}(x, \theta)}{\partial \theta^{2}}+E(x) I(x) \frac{\partial^{4} \mathrm{Y}(x, \theta)}{\partial \theta^{4}}
$$

Analysis of the basic frequency of guide mechanism, the Lanczos method or vector iteration method can be used. However, the impact factors are so many and the research model is more complex. When the system does not require quantitative calculations but only theoretically qualitative analysis, it is possible to refer to the model of Figure2, the relationship between fundamental frequency and dynamic parameters was studied applying Rayleigh iterative method. The basic frequency of guide mechanism can be calculated as follows:

$\frac{\int_{\gamma} E(x) I(x) Y^{\prime 2}(x) d x}{\sum_{i=1}^{n} m_{i} Y^{2}\left(x_{i}\right)+\rho \int_{\gamma} F(x) Y^{2}(x) d x}=\omega^{2}$

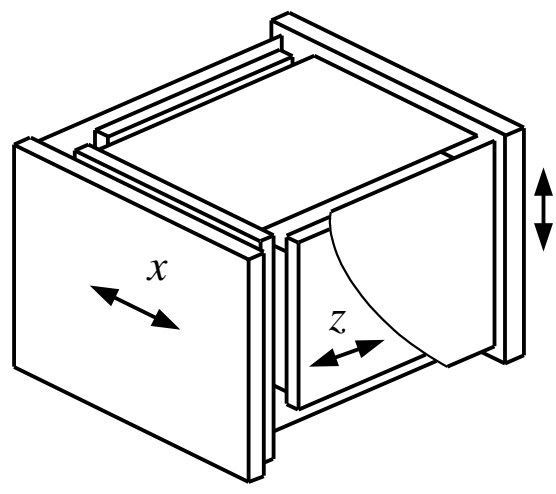

Figure 1.The structure of 3D analogue probe

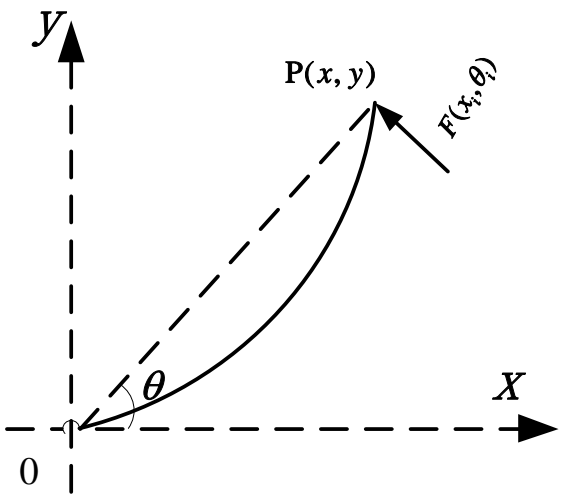

Figure 2. The kinematical analysis of guide mechanism

The above formula can be rewritten as follows:

$\left(\omega_{0}^{2}\right)^{-1}=\frac{\rho \int_{\gamma} F(x) Y^{2}(x) d x}{\int_{\gamma} E(x) I(x) Y^{\prime 2}(x) d x}$

$$
\left(\omega_{i}^{2}\right)^{-1}=\frac{\sum_{i=1}^{n} m_{i} Y^{2}\left(x_{i}\right)}{\int_{\gamma} E(x) I(x) Y^{\prime 2}(x) d x} \quad i=(1,2 ; ; n,
$$

In above formula: $\omega_{\text {is }}$ basic frequency of probe, $Y\left(x_{i}\right)$ is corresponding deflection of the No. $i$ concentrated load. Where, $\omega_{0}$ is assumed that there is no concentrated load and only consider the influence of the basic frequency of the probe. $\omega_{i}$ is assumed that there is no distributed load and only consider the basic frequency of the No. $i$ in total of $n_{\text {concentrated load. }}$

From the formula (2) (3)can be obtained that the effect of concentrated load on the fundamental frequency of the probe is nonlinear. Furthermore, the effect of $m_{i}$ on frequency depends not only on the magnitude of each load, but also on their distribution. The formula(2)can be rewritten as follows: 


$$
\left(\frac{\partial \omega}{\partial \omega_{i}}\right)^{2}=\frac{\sum_{\substack{j \neq i \\ j=0}}^{n} \omega_{j}^{2}\left(\prod_{i=0}^{n} \omega_{i}^{2}\right)-\left(\prod_{\substack{j \neq i \\ j=0}}^{n} \omega_{j}^{2}\right) \sum_{i=0}^{n}\left(\prod_{\substack{j \neq i \\ j=0}}^{n} \omega_{j}^{2}\right)}{-\left[\sum_{i=0}^{n}\left(\prod_{j \neq i}^{n} \omega_{j}^{2}\right)\right]^{2}}
$$

From the above equation, it can be obtained that although the concentrated loads are the same size, due to the different distribution location will also have different effects on the measurement.

\section{Sensitivity Analysis on Influence of Basic Frequency}

In order to illustrate the analytical method and to simplify the research process, the coordinate guide system are equipped with drive mechanism, sensing unit and force balance device, the above equation (3) can be developed as follows:

$$
\begin{aligned}
& \left(\omega^{2}\right)^{-1}=\left(\omega_{0}^{2}\right)^{-1}+\left(\omega_{1}^{2}\right)^{-1}+\left(\omega_{2}^{2}\right)^{-1}+\left(\omega_{3}^{2}\right)^{-1}=\frac{\omega_{1}^{2} \omega_{2}^{2} \omega_{3}^{2}+\omega_{0}^{2} \omega_{2}^{2} \omega_{3}^{2}+\omega_{0}^{2} \omega_{1}^{2} \omega_{3}^{2}+\omega_{0}^{2} \omega_{1}^{2} \omega_{2}^{2}}{\left(\omega_{0} \omega_{1} \omega_{2} \omega_{3}\right)^{2}} \\
& \omega^{2}=\frac{\left(\omega_{0} \omega_{1} \omega_{2} \omega_{3}\right)^{2}}{\omega_{1}^{2} \omega_{2}^{2} \omega_{3}^{2}+\omega_{0}^{2} \omega_{2}^{2} \omega_{3}^{2}+\omega_{0}^{2} \omega_{1}^{2} \omega_{3}^{2}+\omega_{0}^{2} \omega_{1}^{2} \omega_{2}^{2}} \\
& \frac{\partial \omega^{2}}{\partial \omega_{1}^{2}}=\frac{\omega_{0}^{4} \omega_{2}^{4} \omega_{3}^{4}}{\left(\omega_{1}^{2} \omega_{2}^{2} \omega_{3}^{2}+\omega_{0}^{2} \omega_{2}^{2} \omega_{3}^{2}+\omega_{0}^{2} \omega_{1}^{2} \omega_{3}^{2}+\omega_{0}^{2} \omega_{1}^{2} \omega_{2}^{2}\right)^{2}} \\
& \frac{\partial \omega^{2}}{\partial \omega_{2}^{2}}=\frac{\omega_{0}^{4} \omega_{1}^{4} \omega_{3}^{4}}{\left(\omega_{1}^{2} \omega_{2}^{2} \omega_{3}^{2}+\omega_{0}^{2} \omega_{2}^{2} \omega_{3}^{2}+\omega_{0}^{2} \omega_{1}^{2} \omega_{3}^{2}+\omega_{0}^{2} \omega_{1}^{2} \omega_{2}^{2}\right)^{2}} \\
& \frac{\partial \omega^{2}}{\partial \omega_{3}^{2}}=\frac{\omega_{0}^{4} \omega_{1}^{4} \omega_{2}^{4}}{\left(\omega_{1}^{2} \omega_{2}^{2} \omega_{3}^{2}+\omega_{0}^{2} \omega_{2}^{2} \omega_{3}^{2}+\omega_{0}^{2} \omega_{1}^{2} \omega_{3}^{2}+\omega_{0}^{2} \omega_{1}^{2} \omega_{2}^{2}\right)^{2}}
\end{aligned}
$$

Because of $\frac{\partial \omega^{2}}{\partial \omega_{i}}=\frac{\partial \omega^{2}}{\partial \omega_{i}^{2}} \square \frac{\partial \omega_{i}^{2}}{\partial \omega_{i}}$, then:

$$
\begin{aligned}
& \omega_{i}^{2}=\frac{\int_{\gamma} I(x) E(x) Y^{\prime 2}(x) d x}{Y^{2}\left(x_{i}\right) m_{i}} \\
& \frac{\partial \omega_{i}^{2}}{\partial \omega_{i}}=-\frac{\int_{\gamma} I(x) E(x) Y^{\prime 2}(x) d x}{Y^{2}\left(x_{i}\right) m_{i}}=-\frac{\omega_{i}^{2}}{m_{i}}
\end{aligned}
$$

$\frac{\partial \omega^{2}}{\partial \omega_{1}}=-\frac{\omega_{1}^{2}}{m_{1}} \cdot \frac{\omega_{0}^{4} \omega_{2}^{4} \omega_{3}^{4}}{\left(\omega_{1}^{2} \omega_{2}^{2} \omega_{3}^{2}+\omega_{0}^{2} \omega_{2}^{2} \omega_{3}^{2}+\omega_{0}^{2} \omega_{1}^{2} \omega_{3}^{2}+\omega_{0}^{2} \omega_{1}^{2} \omega_{2}^{2}\right)^{2}}$

$\frac{\partial \omega^{2}}{\partial \omega_{2}}=-\frac{\omega_{2}^{2}}{m_{2}} \frac{\omega_{0}^{4} \omega_{1}^{4} \omega_{3}^{4}}{\left(\omega_{1}^{2} \omega_{2}^{2} \omega_{3}^{2}+\omega_{0}^{2} \omega_{2}^{2} \omega_{3}^{2}+\omega_{0}^{2} \omega_{1}^{2} \omega_{3}^{2}+\omega_{0}^{2} \omega_{1}^{2} \omega_{2}^{2}\right)^{2}}$

$$
\frac{\partial \omega^{2}}{\partial \omega_{3}}=-\frac{\omega_{3}^{2}}{m_{3}} \frac{\omega_{0}^{4} \omega_{1}^{4} \omega_{2}^{4}}{\left(\omega_{1}^{2} \omega_{2}^{2} \omega_{3}^{2}+\omega_{0}^{2} \omega_{2}^{2} \omega_{3}^{2}+\omega_{0}^{2} \omega_{1}^{2} \omega_{3}^{2}+\omega_{0}^{2} \omega_{1}^{2} \omega_{2}^{2}\right)^{2}}
$$




$$
\begin{aligned}
\frac{\partial \omega^{2}}{\partial Y\left(x_{i}\right)} & =\frac{\partial \omega^{2}}{\partial \omega_{i}^{2}} \curvearrowleft \frac{\partial \omega_{i}^{2}}{\partial Y\left(x_{i}\right)} \\
\frac{\partial \omega_{i}^{2}}{\partial Y\left(x_{i}\right)} & =-\frac{\omega_{i}^{2}}{m_{i} Y^{2}\left(x_{i}\right)} \frac{\partial\left(\int_{r} I(x) E(x) Y^{\prime \prime 2}(x) d x\right)}{\partial Y\left(x_{i}\right)}
\end{aligned}
$$

From the above conclusion, not only the concentrated load effects on basic frequency is nonlinear relationship, but also the location of the load of the impact is nonlinear. Because the formula was more complex and more influential factors, so the accuracy of the results is difficult to meet the engineering requirements [5]. Experimental modal analysis is to measure the actual system of excitation and response signals to achieve modal parameter identification. In this paper, the experimental modal analysis method was used to establish the model of the analog probe, in order to obtain the accuracy of the system dynamic parameters.

\section{Experiment Analysis}

In order to verify the theoretical relationship between the concentrated load and its position on the basic frequency of the probe, a self-developed probe was used as the research object. The system is fixed in four points, the material of leaf springs is QBe2, the elastic modulus is $126 \mathrm{GPa}$ and the Poisson's ratio of the material is 0.3 . The test system of basic frequency analysis were mainly composed of excitation source, detection unit, data acquisition system, calculation software and computer and other components. The CAYD108 piezoelectric accelerometer was used in the detection unit, and the frequency measurement range is $0.5 \sim 5000 \mathrm{HZ}$, the sensitivity is $100 \mathrm{PC} / \mathrm{g}$.

The finite element method is applied to modal analysis of the guide mechanism, Table 1 shows the impact on the basic frequency of concentrated load and distribution of the probe . With the increase of concentrated load, the working frequency of probe decreases, this will inevitably lead to poor dynamic performance of the probe. Therefore, in order to improve the basic work of the probe, the concentrated load of probe should be reduced and the load distribution position should be optimized.

Table 1 The effect on basic frequency of several concentrated load to probe

\begin{tabular}{|c|c|c|c|}
\hline & $\begin{array}{c}\text { concentrated } \\
\text { load }(\mathrm{mg})\end{array}$ & $\begin{array}{c}\text { Minimum basic } \\
\text { frequency }(\mathrm{Hz})\end{array}$ & $\begin{array}{c}\text { Maximum basic } \\
\text { frequency }(\mathrm{Hz})\end{array}$ \\
\hline $\begin{array}{c}\text { Just consider the influence of } \\
\text { the guiding mechanism load of probe }\end{array}$ & $m_{\mathrm{D}}=317$ & 63.24 & 97.31 \\
\hline \multirow{2}{*}{$\begin{array}{c}\text { Considering the load of } \\
\text { each mechanism of probe }\end{array}$} & $m_{1}=12 m g$ & 12.75 & 61.12 \\
\cline { 2 - 4 } & $m_{2}=28 m g$ & 13.92 & 46.07 \\
\cline { 2 - 4 } & $m_{3}=51 \mathrm{mg}$ & 13.66 & 27.83 \\
\hline
\end{tabular}

\section{Summary}

In order to improve the accuracy and accuracy of the analog probe, this paper has analyzed the relationship between basic frequency and concentrated load. The results show that the influence of the basic frequency of the concentrated load is not only related to the magnitude of the concentrated load, but also to the location of the concentrated load. In the structural design process of the analog probe, the analysis of the frequency response characteristics on the conditions of concentrated load is an effective means to improve the dynamic performance and precision of the analog probe. In the dynamic simulation of the probe design process, this must be sufficient attention and research and analysis. 


\section{References}

[1] LYON S M, ERICKSON P A, EVANS M S, et al: Prediction of the first modal frequency of compliant mechanisms using the pseudo rigid body model [J]. Journal of mechanical Design, Transaction of the ASME, 2009, 121(2):309.

[2] Saxena A, Ananthasuresh K: On a Optimal Property of compliant Topologies [J]. Structure and Multidisciplinary Optimization, 2000, 19:36.

[3] Huai-bo QIANG, Jian-hua WANG. A Design of Three Dimensional Analog Probe Based on Flexure Hinges [J]. Modern Electronic Technology, 2007, 24(1):102.

[4] Elmustafa A A. Lagally Max G. Flexural hinge guided motion stage for precision machining: finite element simulations [J ] . Precision Eng, 2001, 25:77 - 81.

[5] Xin-ming MU, Jian-hua WANG. Mechanical Model for Elastic Guide ways of Parallel Springs [J]. Nanotechnology and Precision Engineering, 2005, 3(4):277.

[6] PingLI,JianhuaWANG,XiaoliLI. Study on Factors of Influencing Inductive Probe CriticalFrequency[J]. Hydromechatronics Engineering, 2006, 10(5):149.

[7] Haiyan LI, Xianmin ZHANG. Research on the Actuation Characteristic of a Large2Def lection Compliant Mechanism [J].Mechanical Scienceand Technology, 2014, 23(9):1041.

[8] Trull,Stephen James, McMurtry.positioning machine: England, US5101548 [P].2003-3-27.

[9] Elmustafa A A. Lagally Max G. Flexural - hinge guided motion nanopositioner stage for precision machining: finite element simulations [J ] . Precision Eng, 2001, 25: 77 - 81.

[10] Hua-wei WANG, Yue-qing YU, Wen-jing WANG, Li-ying SU. Dynamic Modeling and Frequency Analysis of Compliant Parallel Guided Mechanism, 2008, 24(2):42. 